\title{
Erratum to: On Galvin's theorem for compact Hausdorff right-topological semigroups with dense topological centers
}

\author{
Xiongping Dai ${ }^{1, *} \&$ Hailan Liang ${ }^{2}$ \\ ${ }^{1}$ Department of Mathematics, Nanjing University, Nanjing 210093, China; \\ ${ }^{2}$ Department of Mathematics, Fuzhou University, Fuzhou 350116, China \\ Email: xpdai@nju.edu.cn, hailan.liang@smail.nju.edu.cn
}

Erratum to: Science China Mathematics, December 2017 Vol. 60 No. 12: 2421-2428, doi: $10.1007 / \mathrm{s} 11425-016-9139-1$

Citation: Dai X P, Liang H L. Erratum to: On Galvin's theorem for compact Hausdorff right-topological semigroups with dense topological centers. Sci China Math, 2019, 62: 2631-2632, https://doi.org/ $10.1007 / \mathrm{s} 11425-018-1601-4$

In the third paragraph on p. 2421 and in Theorem $1^{\prime}$ of [3] it is wrongly assumed that "the multiplication on a topological semigroup $T$ can be extended to a right continuous multiplication on $\beta T$ "; in other words, we wrongly assume that " $\beta T$ is a compact Hausdorff right-topological semigroup for any topological semigroup $T$ ". So using [3, Theorem 1] we can only obtain the following two theorems in place of [3, Theorem $1^{\prime}$ ], where $X^{\prime}$ denotes the limit-point set of a space $X$ and $J(E)$ denotes the set of all idempotents in a compact Hausdorff right-topological semigroup $E$.

Firstly by [3, Theorem 1] and [6] we can obtain the following instead of [3, Theorem $\left.1^{\prime}\right]$.

Theorem 1' (See [1, Lemma 2.1] and [4, Theorem 3.3] for I and II). Let $T$ be a discrete semigroup. Then the following statements hold:

I. Let $v \in(\beta T)^{\prime} \cap J(\beta T)$ and let $\left\{V_{n}\right\}_{n=1}^{\infty}$ be a sequence of neighborhoods of $v$ in $\beta T$. Then there exists a 1-1 map $\tau_{.}: \mathbb{N} \rightarrow T$ such that $F P\left(\left\langle\tau_{n}\right\rangle_{n=k}^{\infty}\right) \subseteq V_{k}$ for all $k \geqslant 1$. In particular, any neighborhood of $v \in(\beta T)^{\prime} \cap J(\beta T)$ contains an infinite IP-set in $T$.

IIa. For any IP-set $F P\left(\left\langle t_{n}\right\rangle_{n=1}^{\infty}\right)$ in $T$, there exists a $v \in J(\beta T)$ such that $v \in \bigcap_{k=1}^{\infty} \operatorname{cls}_{\beta T} F P\left(\left\langle t_{n}\right\rangle_{n=k}^{\infty}\right)$.

IIb. Let $H$ be a subsemigroup of $T$ such that $H^{\prime}$ is a non-empty subsemigroup of $\beta T$. Then for any infinite IP-set $A \subseteq H$, there exists an idempotent $v \in(\beta T)^{\prime} \cap \operatorname{cls}_{\beta T} A$.

III. If $T$ is weakly left cancelable, then $(\beta T)^{\prime}$ is T-invariant and so $(\beta T)^{\prime}$ is a left ideal in $\beta T$.

Let $G$ be a Hausdorff topological group with continuous multiplication

$$
\lambda: G \times G \stackrel{(t, x) \mapsto t x}{\longrightarrow} G
$$

\footnotetext{
* Corresponding author
} 
and with identity $\mathbb{1}$. Let $\mathscr{U}_{\mathbb{1}}$ be the neighborhood system of $\mathbb{1}$ in $G$. Let $I=[0,1]$ be endowed with the usual topology. A function $\varphi: G \rightarrow I$ is left uniformly continuous, denoted by $F_{\text {luc }}(G)$, if and only if given $\varepsilon>0$ there exists a $U \in \mathscr{U}_{\mathbb{1}}$ such that $|\varphi(x)-\varphi(t x)|<\varepsilon$ for all $x \in G$ and $t \in U$. Define the evaluation map

$$
e_{\mathrm{luc}}: G \stackrel{x \mapsto(\varphi(x))_{\varphi \in F_{\mathrm{luc}}(G)}}{\longrightarrow} I^{F_{\mathrm{luc}}(G)},
$$

and let $G^{\mathrm{LUC}}=\operatorname{cls}_{I^{F_{\text {luc }}(G)}} e_{\text {luc }}(G)$, which is a compact Hausdorff subspace of the compact product space $I^{F_{\text {luc }}(G)}$.

$G^{\mathrm{LUC}}$ is called the LUC-compactification of $G$, which is a compact Hausdorff right-topological semigroup with respect to the canonical multiplication (see $[2,5,7]$ ). Then by $[3$, Theorem 1] we can obtain the following Theorem $1^{\prime \prime}$.

Theorem 1". Let $G$ be any Hausdorff topological group. Then

(I) Let $v \in\left(G^{\mathrm{LUC}}\right)^{\prime} \cap J\left(G^{\mathrm{LUC}}\right)$ and let $\left\{V_{n}\right\}_{n=1}^{\infty}$ be a sequence of neighborhoods of $v$ in $G^{\mathrm{LUC}}$. Then there exists a 1-1 map $\tau .: \mathbb{N} \rightarrow G$ such that $F P\left(\left\langle\tau_{n}\right\rangle_{n=k}^{\infty}\right) \subseteq V_{k}$ for all $k \geqslant 1$. In particular, any neighborhood of $v \in\left(G^{\mathrm{LUC}}\right)^{\prime} \cap J\left(G^{\mathrm{LUC}}\right)$ contains an infinite IP-set in $G$.

(IIa) For any IP-set $F P\left(\left\langle t_{n}\right\rangle_{n=1}^{\infty}\right)$ in $G$, there exists a $v \in J\left(G^{\mathrm{LUC}}\right)$ such that

$$
v \in \bigcap_{k=1}^{\infty} \operatorname{cls}_{G^{\mathrm{LUC}}} F P\left(\left\langle t_{n}\right\rangle_{n=k}^{\infty}\right) .
$$

(IIb) Let $H$ be a subsemigroup of $G$ such that $H^{\prime}$ is a non-empty subsemigroup of $G^{\mathrm{LUC}}$. Then for any infinite IP-set $A \subseteq H$, there exists an idempotent $v \in\left(G^{\mathrm{LUC}}\right)^{\prime} \cap \operatorname{cls}_{G^{\mathrm{LUC}}} A$.

(III) $\left(G^{\mathrm{LUC}}\right)^{\prime}$ is $G$-invariant and so $\left(G^{\mathrm{LUC}}\right)^{\prime}$ is a left ideal in $G^{\mathrm{LUC}}$.

\section{References}

1 Bergelson V, Hindman N. Some topological semicommutative van der Waerden type theorems and their combinatorial consequences. J Lond Math Soc (2), 1992, 45: 385-403

2 Berglund J F, Junghenn H D, Milnes P. Analysis on Semigroups. New York: Wiley, 1989

3 Dai X P, Liang H L. On Galvin's theorem for compact Hausdorff right-topological semigroups with dense topological centers. Sci China Math, 2017, 60: 2421-2428

4 Ellis D, Ellis R, Nerurkar M. The topological dynamics of semigroup actions. Trans Amer Math Soc, 2001, 353: $1279-1320$

5 Flor P. Über eine Kompaktifizierung topologischer Gruppen. J Reine Angew Math, 1967, 228: 193-198

6 Hindman N, Strauss D. Algebra in the Stone-Čech Compactification: Theory and Applications. Berlin: Walter de Gruyter, 1998

7 Knapp A W. Decomposition theorem for bounded uniformly continuous functions on a group. Amer J Math, 1967, 88: 902-914

The online version of the original article can be found at doi: 10.1007/s11425-016-9139-1 